\title{
Optimal design of the transversely vibrating Euler-Bernoulli beams segmented in the longitudinal direction
}

\author{
VEYSEL ALKAND \\ Department of Mechanical Engineering, Pamukkale University, 20070 Pamukkale, Denizli, Turkey \\ e-mail: alkanveysel@gmail.com
}

MS received 1 June 2018; revised 10 December 2018; accepted 4 January 2019; published online 3 April 2019

\begin{abstract}
In this study, optimal design of the transversely vibrating Euler-Bernoulli beams segmented in the longitudinal direction is explored. Mathematical formulation of the beams in bending vibration is obtained using transfer matrix method, which is later coupled with an eigenvalue routine using the "fmincon solver" provided in Matlab Optimization Toolbox. Characteristic equations, namely frequency equations, for determining natural frequencies of the segmented beams for all end conditions are obtained and for each case, square of this equation is selected as a fitness function together with constraints. Due to the explicitly unavailable objective functions for the natural frequencies as a function of segment length and volume fraction of the materials, especially for the beams made of a large number of segments, initially, prescribed value is assumed for the natural frequency and then the variables minimizing objective function and satisfying the constraints are searched. Clamped-free, clamped-clamped, clamped-pinned and pinned-pinned boundary conditions are considered. Among the end conditions, maximum increment in the fundamental natural frequency is more pronounced for the case of clamped-clamped end condition and for this case, maximum increment up to $17.3274 \%$ is attained. Finally, the beam configurations maximizing fundamental natural frequencies will be presented.
\end{abstract}

Keywords. Segmented beam; bending vibration; natural frequencies; optimization.

\section{Introduction}

Nowadays, powder metallurgy has become an important area for engineering, especially in terms of production of structural elements such as beams, columns and plates. Slender beams are the structural members that are widely used in engineering areas such as mechanical, civil, marine and aerospace. Natural frequency is one of their most important dynamic characteristics. It is known that a resonance phenomenon occurs when the main frequencies (i.e., working frequencies) of the structure or systems are equal to or very close to their natural frequencies. That is to say, all objects resonate at their natural frequency when excited. This can cause catastrophic failures in structures, machines and components. One way to solve this problem is to raise the natural frequency enough outside the working range or out of the excitation range. On the other hand, functionally graded materials are the special materials that can be characterized by the variation in composition and structure gradually over the volume in a continuous or piecewise manner, resulting in corresponding changes in the properties of the material such as elastic modulus and density. The concept for functionally graded materials is to make a composite material by varying the microstructure from one material to another material with a specific gradient. This enables the material to have the best of both materials. Therefore, these special materials can be used for specific function and applications. For the beam-type structures made of functionally graded material, natural frequencies can be controlled in a desired manner, i.e., so as to avoid resonance events, and it can be said that design of the beams by controlling their vibration frequencies has been of great interest.

As far as uniform beams are considered, there are many studies available about beam vibration in bending. These problems are best solved using analytical, numerical and experimental methods. Han et al [1] carried out a review study examining four approximate models for a transversely vibrating beam: the Euler-Bernoulli, Rayleigh, shear and Timoshenko models. They presented basic formulations and solutions of the models and they summarize similarities and differences of the theories. In addition, there have been extensive studies concerning optimization of the transversely vibrating beam structures in open literature. This is not the case for the beam made of functionally graded materials, especially in a piecewise manner, i.e., the beam segmented in the longitudinal direction. The number of studies available in literature is rather limited. Application of the finite element to this problem can be found in [2] and [3]. It is mentioned that finite elements with cubic polynomial approximation for the displacement will not 
guarantee obtaining of global optimal solutions. Rather, the general solution to the fourth-order differential equation that governs the vertical beam deflections should be used as a displacement field to formulate the finite element modelling of the beams. Lake and Mikulas [4] presented a study regarding the lateral buckling and vibration of compressively loaded column whose cross-section is piecewise constant along its length. In the case of natural vibration of columns without axial load, they considered two cases. In the first case, mass and bending stiffness of the cross-section are proportional, and in the second one the mass is proportional to the cube root of the bending stiffness. As far as fundamental vibration frequency is concerned, from the parametric studies, they discerned that in the former case, a column with piecewise constant cross-section is less efficient than a uniform column, whereas, in the latter case, the column with piecewise constant cross-section is more efficient than the uniform column, based on either fundamental vibration frequency or buckling load. Kukla and Rychlewska [5] conducted free vibration analysis of the axially functionally graded clamped-clamped two-segmented beams. This analysis is based on the approximation of the functionally graded beam by piecewise exponentially varying geometrical and material properties. They extended their studies by considering a simply supported beam with an arbitrary number of segments [6]. Some numerical examples are tabulated and they mentioned that the accuracy of the eigenfrequencies improves as the number of segments increases. Li et al [7] presented free vibration analysis of cantilevered tall structures under various axial loads. The beam considered is divided into several segments in such a manner that the segments are divided appropriately and the distribution of flexural stiffness and mass in each segment may match accurately or approximately the one described in a continuous manner. They used transfer matrix method to solve the eigenvalue problems. Goupee and Vel [8] proposed a methodology for optimizing natural frequencies of a functionally graded beam with variable volume fraction of the constituent materials along length and height directions. They used a piecewise bi-cubic interpolation of volume fraction values specified at a finite number of grid points, and applied genetic algorithm method to find the optimum designs. They considered three problems: finding material distributions maximizing each of the first three natural frequencies of the beam, minimizing mass of the beam while constraining its natural frequencies lying outside certain prescribed frequency bands and minimizing the mass of the beam by simultaneously optimizing its thickness and material distribution such that the fundamental frequency is greater than a prescribed value. Finally, they pointed out that material distribution has a significant influence on natural frequencies. Kai-yuan et al [9] proposed a modified step-reduction method to investigate dynamic response of the Bernoulli-Euler beams with arbitrary nonhomogeneity and variable cross-section under arbitrary loads. The method requires discretizing the space domain into a number of elements and each element is treated as a homogeneous one with uniform thickness. They used initial parameters method for the analytical solution of the problem and considered both free and force vibration cases. As an example of free vibration case, they considered a stepped beam (a two-element stepped beam) and derived frequency equation. Zhou and Ji [10] investigated dynamic characteristics of a beam with continuously distributed spring-mass. They thought that this condition resembles a structure occupied by a crowd of people. Separating the attached spring-mass from the beam segment and considering the actions of the spring-mass on the beam, the governing differential equations of the beam segment and the distributed spring-mass on the segment are given. Then, the eigenvalue equations for the beam with different end conditions are obtained using the transfer matrix method.

In the present study, optimal design of the segmented Euler-Bernoulli beams in transverse vibrations is explored using the "fmincon" solver provided in Matlab Optimization Toolbox. In the first place, characteristic equations, namely frequency equations, for determining natural frequencies for all boundary conditions in the dimensionless forms are obtained using the transfer matrix method. Then, for each case, square of this equation is selected as an objective function along with mass and length constraints. However, it is very difficult to explicitly obtain objective function for the natural frequencies as a function of volume fraction and segment length. Therefore, for each boundary condition, prespecified natural frequencies chosen to be slightly larger than those obtained from uniform beams given in table 1 are assumed and then design variables satisfying constraints are explored. The design variables are, on the other hand, volume fraction and length of the segments. Clamped-free (cf), clamped-clamped (cc), clamped-pinned (cp) and pinned-pinned (pp)-type boundary conditions are considered. Finally, beam configurations maximizing fundamental natural frequencies will be determined. On the other hand, as far as beam design in bending vibration in literature is considered, it can be pointed out that the present study offers an objective function formulation different from the one discussed earlier. The fitness function formulation coupled with eigenvalue routine is the merit of this study and can also be applied to any other problem, which includes transcendental functions in particular.

\section{Mathematical formulation for the segmented beams}

A true and robust optimization routine strongly depends on the mathematical modelling of the problem. Hence, first, an exact structural analysis of the beam should be carried out. Figure 1 shows elastic and slender beam structures: 
Table 1. Characteristic equations for the natural frequencies of the beams.

\begin{tabular}{lccc}
\hline End type & Boundary conditions & Characteristic equation & $\widehat{\omega}$ \\
\hline cf & $Y_{1}=\theta_{1}=0$ and $M_{N_{s}}=F_{N_{s}}=0$ & $T_{33} T_{44}-T_{34} T_{43}=0$ & 3.5160 \\
cc (whole span) & $Y_{1}=\theta_{1}=0$ and $Y_{N_{s}}=\theta_{N_{s}}=0$ & $T_{13} T_{24}-T_{14} T_{23}=0$ & 22.3733 \\
cc (half span) & $Y_{1}=\theta_{1}=0$ and $\theta_{N_{s}}=F_{N_{s}}=0$ & $T_{23} T_{44}-T_{24} T_{43}=0$ & 22.3733 \\
cp & $Y_{1}=\theta_{1}=0$ and $Y_{N_{s}}=M_{N_{s}}=0$ & $T_{13} T_{34}-T_{14} T_{33}=0$ & 15.4182 \\
pp (whole span) & $Y_{1}=M_{1}=0$ and $Y_{N_{s}}=M_{N_{s}}=0$ & $T_{12} T_{34}-T_{14} T_{32}=0$ & 9.8696 \\
pp (half span) & $Y_{1}=M_{1}=0$ and $\theta_{N_{s}}=F_{N_{s}}=0$ & $T_{22} T_{44}-T_{24} T_{42}=0$ & 9.8696 \\
\hline
\end{tabular}

\begin{tabular}{|c|c|}
\hline $\mathrm{V}_{\mathrm{A}}=50 \%$ & $V_{B}=50 \%$ \\
\hline & 1 \\
\hline
\end{tabular}

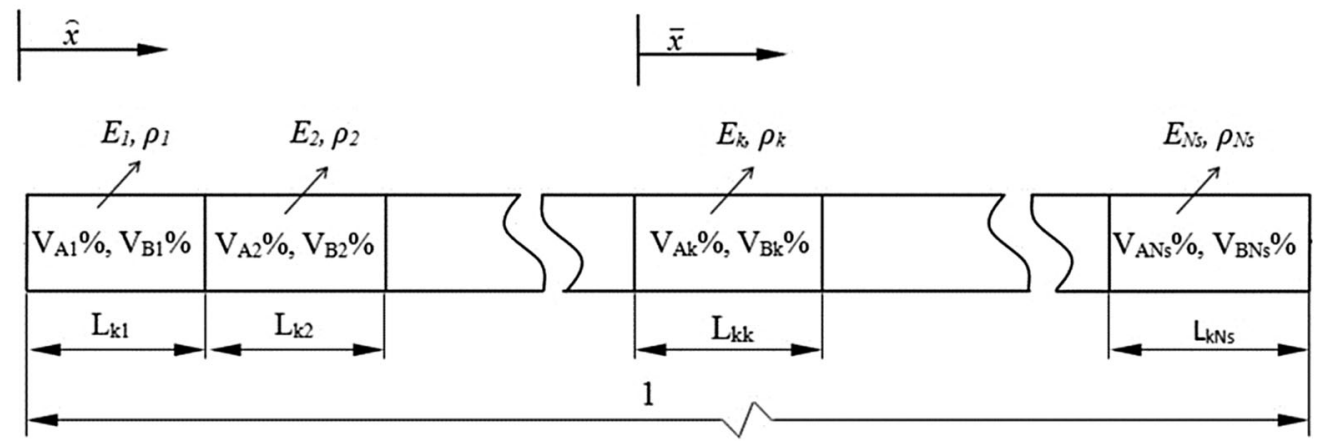

Figure 1. General view of the one-segment and multi-segmented beam with $N_{s}$ segments.

uniform baseline beam consisting of one segment and multi-segmented beam consisting of predetermined number of uniform elements $\left(N_{s}\right)$ [11].

Based on the Euler-Bernoulli beam theory, the governing differential equation of the free transverse vibration of the $k^{\text {th }}$ element is described by the following equation, where $Y$ is the bending deflection, $x$, axial coordinate, $E_{k}$, $I_{k}, A_{k}, L_{k}$ and $\rho_{k}$ are Young's modulus, moment of inertia, cross-section, length and mass density of the $k^{\text {th }}$ segment, respectively, and $\omega$ is the natural frequency of the beam:

$$
E_{k} I_{k} \frac{\partial^{4} Y}{\partial x^{4}}-\rho_{k} A_{k} \omega^{2} Y=0, \quad x_{k} \leq x \leq x_{k+1} .
$$

The various dimensionless quantities denoted by ${ }^{\wedge}$ are defined as follows:

$$
\begin{aligned}
& \widehat{E}_{k}=\frac{E_{k}}{E}, \widehat{I}_{k}=\frac{I_{k}}{I}, \widehat{A}_{k}=\frac{A_{k}}{A}, \widehat{\rho}_{k}=\frac{\rho_{k}}{\rho}, \widehat{Y}=\frac{Y}{L}, \widehat{x}=\frac{x}{L}, \\
& \widehat{L}_{k}=\frac{L_{k}}{L}, \widehat{\omega}=\omega L^{2} \sqrt{\frac{\rho A}{E I}}
\end{aligned}
$$

where $E, I, A, \rho$ and $L$ are the variables of the reference baseline design beam structure defined as having uniform mass and stiffness distributions and consisting of only one segment. $\widehat{Y}$ is the dimensionless bending deflection, $\widehat{x}$, the dimensionless axial coordinate and $\hat{\omega}$, dimensionless fundamental natural frequency.
It is assumed that the optimized design will have the same total mass, total length, cross-sectional dimensions, shape and type of material constructions and type of the boundary conditions as those of baseline design. Substituting dimensionless quantities into Eq. (1) leads to the following fourth-order differential equation:

$$
\frac{\partial^{4} \widehat{Y}}{\partial \widehat{x}^{4}}-\lambda^{4} \widehat{Y}=0
$$

where the parameter $\lambda$ is defined as $\lambda=\sqrt{\widehat{\omega} \sqrt{\frac{\widehat{\rho}_{k}}{\hat{E}_{k}}}}$.

Equation (2) must be satisfied in the interval $0 \leq \bar{x} \leq L_{k}$ where $\bar{x}=\widehat{x}-\widehat{x}_{k}$. From the theory of linear differential equations, the solution of Eq. (2) is

$$
\begin{aligned}
\hat{Y}(\bar{x})= & A_{1} \sin (\lambda \bar{x})+A_{2} \cos (\lambda \bar{x}) \\
& +A_{3} \sinh (\lambda \bar{x})+A_{4} \cosh (\lambda \bar{x})
\end{aligned}
$$

where $A_{i}(i=1,2,3,4)$ are unknown constants determined from boundary conditions. On the other hand, it is known that the Euler-Bernoulli beam theory neglects the effects of both rotational inertia and shear deformation. However, for the slender beams, the theory gives satisfactory results [1]. 
A transfer matrix method is applied herein to establish the eigenvalue equation for the transverse vibration of the segmented beam. Detailed information about the transfer matrix method can be found in [12]. For the Euler-Bernoulli beam, the relation between dimensionless displacement $(\widehat{Y})$, slope $(\theta)$, bending moment $(\widehat{M})$ and the shear force $(\widehat{F})$ can be written as

$$
\widehat{Y}=\widehat{Y}, \theta=\widehat{Y}^{\prime}, \widehat{M}=\widehat{E}_{k} \widehat{I}_{k} \widehat{Y}^{\prime \prime} \text { and } \widehat{F}=\widehat{E}_{k} \widehat{I}_{k} \widehat{Y}^{\prime \prime \prime} .
$$

The coefficients $A_{i}$ of Eq. (3) can be expressed in terms of the state variables using Eq. (4). At both nodes of the $k^{\text {th }}$ segment, the following relation can be obtained:

$$
\begin{aligned}
\left\{\begin{array}{c}
\hat{Y}_{k+1} \\
\theta_{k+1} \\
\hat{M}_{k+1} \\
\hat{F}_{k+1}
\end{array}\right\}= & {\left[\begin{array}{cccc}
S & T / \lambda & U /\left(\widehat{E}_{k} \lambda^{2}\right) & U /\left(\widehat{E}_{k} \lambda^{3}\right) \\
\lambda V & S & T /\left(\widehat{E}_{k} \lambda\right) & U /\left(\widehat{E}_{k} \lambda^{2}\right) \\
\widehat{E}_{k} \lambda^{2} U & \widehat{E}_{k} \lambda V & S & T / \lambda \\
\widehat{E}_{k} \lambda^{3} T & \widehat{E}_{k} \lambda^{2} U & \lambda V & S
\end{array}\right] } \\
& \left\{\begin{array}{c}
\hat{Y}_{k} \\
\theta_{k} \\
\hat{M}_{k} \\
\hat{F}_{k}
\end{array}\right\}
\end{aligned}
$$

where $S=(c h+c) / 2, \quad T=(s h+s) / 2, \quad U=(c h-c) / 2$, $V=(s h-s) / 2, c h=\cosh (\lambda \nmid), s h=\sinh (\lambda \nmid), c=\cos (\lambda \nmid)$ and $s=\sin (\lambda \nmid)$. These abbreviations are taken from [13].

Successively applying Eq. (5) to all segments of the beam and considering continuity conditions, the state variables at both ends of the beam can be related to each other through a matrix [T] called as the transfer matrix of the Euler-Bernoulli beam components and given as

$$
[T]=\left[T_{n}\right]\left[T_{n-1}\right] \cdots\left[T_{2}\right]\left[T_{1}\right]
$$

Hence, implementing the boundary conditions and considering non-trivial solutions, associated eigenfrequency equations that will be used for constructing objective functions of the design problems can be obtained. Table 1 shows the reference dimensionless fundamental natural frequencies, $(\hat{\omega})$, and the characteristic equations of a onesegment beam on different end conditions, namely baseline beam design, which will be used for comparison. The same results can also be found using segmented beams. In this case, all segments have equal length and each has equal volume fraction of the materials.

\section{Optimization problem formulation for the natural frequencies of the beams}

The beam considered consists of different numbers of segments and each segment is made of two different materials denoted as A and B. Hence, each segment has different material properties (i.e., elastic modulus and mass density) depending on volume fraction of the materials used. In addition, all segments have the same cross-sectional properties (i.e., moment of inertia and cross-sectional dimensions). For prediction of Young's modulus and mass density, the Halpin-Tsai model is used and the following relations under the assumption that no voids are present can be written:

$$
\begin{gathered}
V_{A}(x)+V_{B}(x)=1 \\
E(x)=V_{A}(x) E_{A}+V_{B}(x) E_{B} \\
\rho(x)=V_{A}(x) \rho_{A}+V_{B}(x) \rho_{B}
\end{gathered}
$$

where $V_{A}, E_{A}$ and $\rho_{A}$ are volume fraction, elastic modulus and mass density of the material $A$, respectively. $V_{B}, E_{B}$ and $\rho_{B}$ denote volume fraction, elastic modulus and mass density of the material $B$, respectively.

On the other hand, all natural frequencies obtained from optimization cycle are compared to those obtained from baseline designs having uniform material properties and constructed from the same type of composite material with equal volume fraction of its constituents, that is $V_{A}=V_{B}=50 \%$. Hence, Young's modulus and mass density of baseline design can be calculated as

$$
\begin{gathered}
E=\frac{\left(E_{A}+E_{B}\right)}{2} \\
\rho=\frac{\left(\rho_{A}+\rho_{B}\right)}{2}
\end{gathered}
$$

and for each segment of the beam, Young's modulus and mass density can be determined from the following relations:

$$
\begin{aligned}
& \hat{\rho}_{k}=\frac{\rho_{A} V_{(A, k)}+\rho_{B} V_{(B, k)}}{\rho}, \quad k=1 \text { to } N_{s} \\
& \hat{E}_{k}=\frac{E_{A} V_{(A, k)}+E_{B} V_{(B, k)}}{E}, \quad k=1 \text { to } N_{s}
\end{aligned}
$$

in which $\hat{\rho}_{k}$ and $\hat{E}_{k}$ are the dimensionless mass density and Young's modulus of the $k^{\text {th }}$ segment, respectively. $V_{(A, k)}$ and $V_{(B, k)}$ are defined as the volume fraction of the materials $A$ and $B$ in the $k^{\text {th }}$ segment, respectively.

It is also noted that the non-dimensional mass of the beam $\left(\hat{M}_{s}\right)$ can be calculated using Eq. (14), which is the equality constraint of the optimization problem. It implies that the optimized beam has the same total mass as its baseline beam structure.

$$
\hat{M}_{s}=\frac{M_{s}}{M}=\sum_{k=1}^{N_{s}} \hat{\rho}_{k} \hat{L}_{k}=1
$$

where $M$ is the total mass of the baseline beam, $M_{s}$, total mass of the optimized segmented beam structure and $\hat{L}_{k}$, non-dimensional length of the $k^{\text {th }}$ segment. 
On the other hand, it is well known that Young's modulus and mass density, both of which are functions of volume fractions of the materials and segment length, are some of the main factors affecting the natural frequencies of the beam; $\hat{\omega}$, the fitness function, should be explicitly obtained as a function of these. This is not the case for the present problem, especially for the beam structures consisting of higher number of segments, as mentioned in the preceding section. Therefore, $\hat{\omega}$ is assumed to take predefined values depending on the end conditions. Its value is somewhat bigger than the one given in table 1 for each end condition. After assigning its value, then, $V$ (volume fraction of the materials $A$ or $B$ ) and $\widehat{L}_{k}$ that minimize square of the characteristic equation are searched during the optimization cycle. If it exists, squaring the characteristic equation ensures that the minimum value would be zero $[14,15]$. Also, for realistic beam design in terms of production, side constraints are present and upper and lower limits should be prescribed. Finally, the present optimization problem can be stated as follows:

find

$$
\mathbf{x}=\left\{\begin{array}{c}
x_{1} \\
x_{1} \\
\cdot \\
\cdot \\
\cdot \\
x_{n}
\end{array}\right\}
$$

that minimizes

$$
\left[\operatorname{characteristic} \text { equation }\left(V, \widehat{L}_{k}\right)\right]^{2}
$$

subject to $h_{1}(\mathbf{x})=\hat{M}_{s}=1$ and

$$
h_{2}(\mathbf{x})=\sum_{k=1}^{N_{s}} \hat{L}_{k}=1
$$

or for symmetric cases

$$
\begin{aligned}
& h_{1}(\mathbf{x})=\hat{M}_{s}=0.5 \quad \text { and } \\
& h_{2}(\mathbf{x})=\sum_{k=1}^{N_{s}} \hat{L}_{k}=0.5
\end{aligned}
$$

side constraints:

$$
\begin{aligned}
& 0 \leq\left(V, \widehat{L}_{k}\right) \leq 1 \quad \text { for whole span } \\
& 0 \leq V \leq 1 \text { and } 0 \leq \widehat{\mathrm{L}}_{\mathrm{k}} \leq 0.5 \text { for half span. }
\end{aligned}
$$

Due to the symmetric conditions, it is possible to consider only half of the beam structures for the pp and cc end conditions. Hence, the total mass and the total length of the beam are reduced to half. It is also noted that using massend length constraints, the number of design variables can be reduced for the sake of reducing CPU time.
There is no single method for solving such a constrained optimization problem stated earlier. Extensive traditional and modern optimization methods have been developed for solving different types of optimization problems. As far as frequency optimization is concerned, there are many studies using different optimization techniques. The modified feasible direction (MFD) method [16], Artificial Bee Colony Algorithm (ABCA) [17] and teaching-learning-based optimization (TLBO) [18] are some examples of the techniques. Detailed information about optimization techniques can be found in the books $[19,20]$. On the other hand, in this study, "fmincon solver" coupled with an eigenvalue routine is used as a tool for solving the problem stated earlier. The flowchart of the optimization procedure is given in figure 2.

Matlab Optimization Toolbox provides functions for finding parameters that minimize or maximize objectives while satisfying constraints. The toolbox includes solvers for linear and nonlinear programming; "fmincon" is a nonlinear programming solver using four algorithm options. They are "interior-point" (default), "trust-regionreflective", "SQP (Sequential Quadratic Programming)" and "active-set". As mentioned in [21], among the fmincon algorithms used, it is recommended that "interior-point" is first used. Reasoning behind this recommendation is that the interior-point algorithm is used for general nonlinear optimization. It is especially useful for large-scale problems

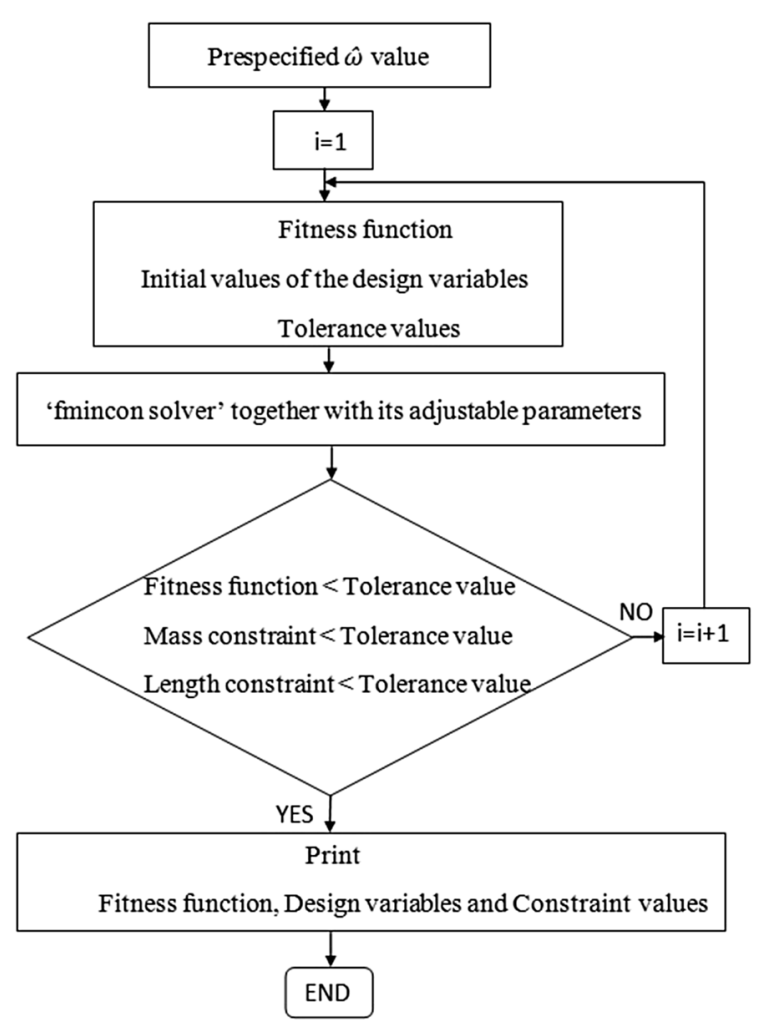

Figure 2. Flowchart of the optimization procedure. 
Table 2. Results of the optimization routine for the beams with clamped-free end conditions for different segment numbers $\left(N_{s}\right)$.

\begin{tabular}{|c|c|c|}
\hline Segment number & Optimization routine parameters & Values \\
\hline \multirow[t]{8}{*}{$N_{s}=2$} & Design variables & $\left(\begin{array}{llll}0.5402 & 0.4597 & 0.5010 & 0.4990\end{array}\right)$ \\
\hline & Fitness function & $1.1135 \mathrm{e}-21$ \\
\hline & Function evaluations & 95 \\
\hline & Mass constraint & 0 \\
\hline & Length constraint & 0 \\
\hline & $\widehat{\omega}$ & 3.6570 \\
\hline & Gain $(\%)$ & 4.0094 \\
\hline & Elapsed time (s) & 1.007590 \\
\hline \multirow[t]{8}{*}{$N_{s}=3$} & Design variables & 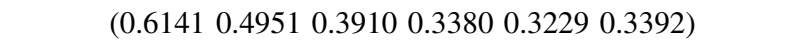 \\
\hline & Fitness function & $4.3144 \mathrm{e}-21$ \\
\hline & Function evaluations & 608 \\
\hline & Mass constraint & 0 \\
\hline & Length constraint & $2.2204 \mathrm{e}-16$ \\
\hline & $\widehat{\omega}$ & 3.9000 \\
\hline & Gain $(\%)$ & 10.9215 \\
\hline & Elapsed time (s) & 1.047910 \\
\hline \multirow[t]{8}{*}{$N_{s}=4$} & Design variables & 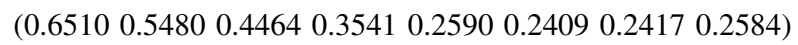 \\
\hline & Fitness function & $7.1175 \mathrm{e}-21$ \\
\hline & Function evaluations & 1764 \\
\hline & Mass constraint & $2.2204 \mathrm{e}-16$ \\
\hline & Length constraint & 0 \\
\hline & $\widehat{\omega}$ & 4.0000 \\
\hline & Gain $(\%)$ & 13.7656 \\
\hline & Elapsed time (s) & 1.351997 \\
\hline
\end{tabular}

Table 3. Results of the optimization routine for the beams with clamped-pinned end conditions for different segment numbers $\left(N_{s}\right)$.

\begin{tabular}{|c|c|c|}
\hline Segment number & Optimization routine parameters & Values \\
\hline \multirow[t]{8}{*}{$N_{s}=2$} & Design variables & (0.6533 0.42470 .32930 .6707$)$ \\
\hline & Fitness function & $6.2082 \mathrm{e}-19$ \\
\hline & Function evaluations & 495 \\
\hline & Mass constraint & 0 \\
\hline & Length constraint & 0 \\
\hline & $\widehat{\omega}$ & 15.7000 \\
\hline & Gain $(\%)$ & 1.8277 \\
\hline & Elapsed time (s) & 1.098915 \\
\hline \multirow[t]{8}{*}{$N_{s}=3$} & Design variables & 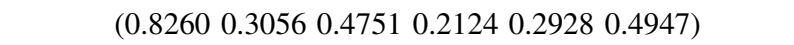 \\
\hline & Fitness function & $2.3717 \mathrm{e}-18$ \\
\hline & Function evaluations & 769 \\
\hline & Mass constraint & 0 \\
\hline & Length constraint & 0 \\
\hline & $\widehat{\omega}$ & 16.5000 \\
\hline & Gain $(\%)$ & 7.0164 \\
\hline & Elapsed time (s) & 1.184472 \\
\hline \multirow[t]{8}{*}{$N_{s}=4$} & Design variables & 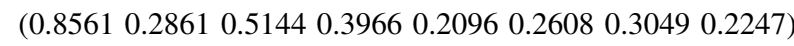 \\
\hline & Fitness function & $9.5957 \mathrm{e}-18$ \\
\hline & Function evaluations & 1410 \\
\hline & Mass constraint & $5.5511 \mathrm{e}-16$ \\
\hline & Length constraint & 0 \\
\hline & $\widehat{\omega}$ & 16.6500 \\
\hline & Gain $(\%)$ & 7.9893 \\
\hline & Elapsed time (s) & 1.615644 \\
\hline
\end{tabular}

that have structure, and tolerates user-defined objective and constraint function evaluation failures. It is based on a barrier function, and optionally keeps all iterations strictly feasible with respect to bounds during the optimization run. The fmincon interior-point algorithm can accept a Hessian function as an input. It can also be possible for the user to 
Table 4. Results of the optimization routine for the beams with pinned-pinned end conditions for different segment numbers $\left(N_{s}\right)$.

\begin{tabular}{|c|c|c|c|}
\hline & $\begin{array}{l}\text { Segment } \\
\text { number }\end{array}$ & $\begin{array}{l}\text { Optimization routine } \\
\text { parameters }\end{array}$ & Values \\
\hline \multirow{8}{*}{$\begin{array}{l}\text { Whole } \\
\text { span }\end{array}$} & \multirow[t]{8}{*}{$N_{s}=4$} & Design variables & 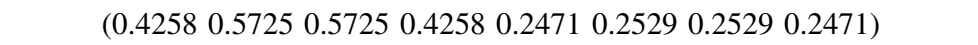 \\
\hline & & Fitness function & $4.222 \mathrm{e}-21$ \\
\hline & & Function evaluations & 259 \\
\hline & & Mass constraint & $2.2204 \mathrm{e}-16$ \\
\hline & & Length constraint & $1.1102 \mathrm{e}-16$ \\
\hline & & $\widehat{\omega}$ & 10.0670 \\
\hline & & Gain $(\%)$ & 2.0001 \\
\hline & & Elapsed time (s) & 1.243094 \\
\hline \multirow[t]{8}{*}{ Half span } & \multirow[t]{8}{*}{$N_{s}=2$} & Design variables & $\left(\begin{array}{lllll}0.4256 & 0.5718 & 0.2456 & 0.2544\end{array}\right)$ \\
\hline & & Fitness function & $7.3143 \mathrm{e}-24$ \\
\hline & & Function evaluations & 145 \\
\hline & & Mass constraint & 0 \\
\hline & & Length constraint & 0 \\
\hline & & $\widehat{\omega}$ & 10.0670 \\
\hline & & Gain $(\%)$ & 2.0001 \\
\hline & & Elapsed time (s) & 1.179807 \\
\hline \multirow[t]{8}{*}{$\begin{array}{l}\text { Whole } \\
\text { span }\end{array}$} & \multirow[t]{8}{*}{$N_{s}=6$} & Design variables & 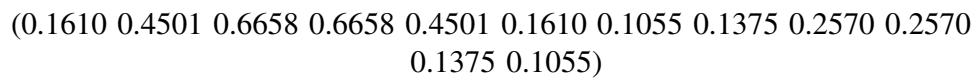 \\
\hline & & Fitness function & $8.8533 \mathrm{e}-18$ \\
\hline & & Function evaluations & 2292 \\
\hline & & Mass constraint & 0 \\
\hline & & Length constraint & 0 \\
\hline & & $\widehat{\omega}$ & 10.3039 \\
\hline & & Gain $(\%)$ & 4.4004 \\
\hline & & Elapsed time (s) & 2.670104 \\
\hline \multirow[t]{8}{*}{ Half span } & \multirow[t]{8}{*}{$N_{s}=3$} & Design variables & $\left(\begin{array}{llllll}0.1597 & 0.4518 & 0.6671 & 0.1055 & 0.1395 & 0.2550\end{array}\right)$ \\
\hline & & Fitness function & $4.5835 \mathrm{e}-17$ \\
\hline & & Function evaluations & 1145 \\
\hline & & Mass constraint & 0 \\
\hline & & Length constraint & 0 \\
\hline & & $\widehat{\omega}$ & 10.3039 \\
\hline & & Gain $(\%)$ & 4.4004 \\
\hline & & Elapsed time (s) & 1.429477 \\
\hline
\end{tabular}

specify the type of Hessian approximation or exact function. Approximation types that users can choose are Broyden-Fletcher-Goldfarb-Shanno (BFGS), Limitedmemory Broyden-Fletcher-Goldfarb-Shanno (LBFGS) and finite difference on gradients. The algorithm satisfies bounds at all iterations, and can recover from "NaN" or "Inf" results. The fmincon solver finds the minimum of constrained nonlinear multi-variable function. It finds the minimum of the problem specified as

$\min _{x} f(x)$ such that

$$
\left\{\begin{array}{c}
c(x) \leq 0 \\
c e q(x)=0 \\
A x \leq b \\
\text { Aeq. }=b e q \\
l b \leq x \leq u b
\end{array}\right\}
$$

where $b$ and beq are vectors, $A$ and $A e q$ are matrices, $c(x)$ and $\operatorname{ceq}(x)$ are functions that return vectors and $f(x)$ is a function that returns a scalar; $x, l b$ and $u b$ can be considered as vectors or matrices. On the other hand, $f(x)$ refers to the objective function, $x$, design vector, $l b$, lower bounds and $u b$, upper bounds of the design variables [21].

\section{Result and discussion}

The segmented beams considered are made of two different materials (e-glass/epoxy) and their material properties are $\rho_{A}=2.54 \mathrm{~g} / \mathrm{cm}^{3}, \rho_{B}=1.27 \mathrm{~g} / \mathrm{cm}^{3}, E_{A}=73 \mathrm{GPa}$ and $E_{B}=$ $4.3 \mathrm{GPa}$ [11]. Four different end conditions are considered: cc, cf, cp and pp. Three different beam structures made of 2, 3 and 4 segments for cf- and cp-type boundary conditions are examined. For simply supported and cc end conditions showing symmetry properties, half-span beams consisting of 1 segment, 2 and 3 segments and corresponding wholespan beams consisting of 2, 4 and 6 segments are also considered. 
As far as manufacturing aspects are considered, rounding numerical values to some extent depending on the usage can be used. As the fmincon parameters, tolerance values for objective function, constraints (mass and length constraints) and design variables are chosen to be $10^{-15}$ (default values are $10^{-6}$ ). Moreover, in the following tables, the gain is the percentage increase in the fundamental natural frequency and it is calculated by comparing the results obtained from the one-segment baseline and multisegmented beams.

Design variables, fitness function, function evaluations, constraints values, dimensionless fundamental natural frequencies, gains and elapsed time are tabulated for different end conditions and segment numbers in the following tables. As mentioned before, the design variables are the segment length and the volume fraction of the materials. Hence, the numbers within the parentheses appearing in the design variables represent

$$
\left(V_{k}, L_{k}\right)=\left(V_{A 1}, V_{A 2}, \ldots, V_{A N_{S}}, L_{k 1}, L_{k 2}, \ldots, L_{k N_{S}}\right) .
$$

As an example, for a three-segmented beam, design variable takes the form

$\mathbf{x}=\left(V_{k}, L_{k}\right)=\left(V_{A 1}, V_{A 2}, V_{A 3}, L_{k 1}, L_{k 2}, L_{k 3}\right)$ in which $V_{A 1}, V_{A 2}$ and $V_{A 3}$ represent volume fraction of the material $A$ of the first, second and third segments and $L_{k 1}, L_{k 2}$ and $L_{k 3}$ refer to the lengths of the first, second and third segments of the beam structure, respectively. This is the case for all design variables appearing in tables 2, 3, 4 and 5 .

In tables 2, 3, 4 and 5, it is observed that for all cases, parallel to an increase in the segment number, dimensionless fundamental natural frequencies increase and at the same time, the gain increases. That is to say, the more the segment number, the higher the natural frequencies obtained. Hence, the maximum non-dimensional natural frequencies are attained for the beam structure with four segments. Therefore, the following results correspond to the beams with four segments. The maximum non-dimensional natural frequency for the cantilevered beam structure is 4.0000 , which represents $13.7656 \%$ optimization gain. It

Table 5. Results of the optimization routine for the beams with clamped-clamped end conditions for different segment numbers $\left(N_{s}\right)$.

\begin{tabular}{|c|c|c|c|}
\hline & $\begin{array}{l}\text { Segment } \\
\text { number }\end{array}$ & $\begin{array}{l}\text { Optimization routine } \\
\text { parameters }\end{array}$ & Values \\
\hline \multirow{8}{*}{$\begin{array}{r}\text { Whole } \\
\text { span }\end{array}$} & \multirow[t]{8}{*}{$N_{s}=4$} & Design variables & 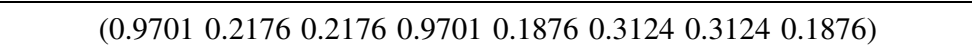 \\
\hline & & Fitness function & $9.7389 \mathrm{e}-18$ \\
\hline & & Function evaluations & 979 \\
\hline & & Mass constraint & 0 \\
\hline & & Length constraint & 0 \\
\hline & & $\widehat{\omega}$ & 26 \\
\hline & & Gain $(\%)$ & 16.2099 \\
\hline & & Elapsed time (s) & 1.285894 \\
\hline \multirow[t]{8}{*}{ Half span } & \multirow{8}{*}{$N_{s}=2$} & Design variables & $\left(\begin{array}{llll}0.9700 & 0.2166 & 0.1881 & 0.3119\end{array}\right)$ \\
\hline & & Fitness function & $6.6589 \mathrm{e}-17$ \\
\hline & & Function evaluations & 473 \\
\hline & & Mass constraint & 0 \\
\hline & & Length constraint & 0 \\
\hline & & $\widehat{\omega}$ & 26 \\
\hline & & Gain $(\%)$ & 16.2099 \\
\hline & & Elapsed time (s) & 1.236247 \\
\hline \multirow[t]{8}{*}{$\begin{array}{r}\text { Whole } \\
\text { span }\end{array}$} & \multirow[t]{8}{*}{$N_{s}=6$} & Design variables & 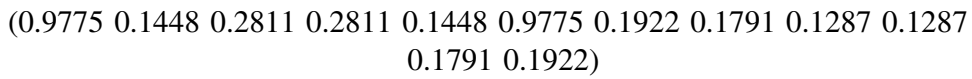 \\
\hline & & Fitness function & $6.8386 \mathrm{e}-17$ \\
\hline & & Function evaluations & 2655 \\
\hline & & Mass constraint & 0 \\
\hline & & Length constraint & 0 \\
\hline & & $\widehat{\omega}$ & 26.25 \\
\hline & & Gain $(\%)$ & 17.3274 \\
\hline & & Elapsed time (s) & 3.049958 \\
\hline \multirow[t]{8}{*}{ Half span } & \multirow[t]{8}{*}{$N_{s}=3$} & Design variables & 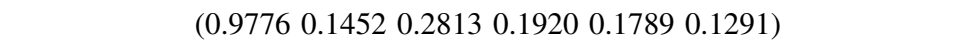 \\
\hline & & Fitness function & $5.9561 \mathrm{e}-16$ \\
\hline & & Function evaluations & 1107 \\
\hline & & Mass constraint & $1.1102 \mathrm{e}-16$ \\
\hline & & Length constraint & 0 \\
\hline & & $\widehat{\omega}$ & 26.25 \\
\hline & & Gain $(\%)$ & 17.3274 \\
\hline & & Elapsed time (s) & 1.443906 \\
\hline
\end{tabular}


is 16.6500 (7.9893\% optimization gain) for the cp, 10.3039 (corresponding to $4.4004 \%$ gain) for the pp and 26.2500 ( $17.3274 \%$ optimization gain) for the cc beams. From the results, it is discerned that the maximum increment in the dimensionless natural frequencies of the beam is observed for the $\mathrm{cc}$ end condition. Every dynamic system (beam, plate, etc.) has stiffness and mass properties. Stiffness comes from potential energy, which is a function of boundary condition. Different boundary conditions introduce different reactions (forces and moments) at the supports, so the stiffness of the vibrating system changes while mass remains the same. Among the end conditions considered, the structure with cc boundary conditions has higher stiffness, which in turns results in higher natural frequencies. Hence, the reason for the maximum increase in the frequency of the beam with fixed-fixed end conditions can be attributed to this fact. Considering the simply supported case, there is a satisfactory agreement between the results obtained from the present study and [2]. At the same time, it should not be forgotten that care must be taken for the increased manufacturing costs. Hence, there should be a balance between the initial design requirements and the beam configurations.

In addition, extensive computer analysis shows that there is no way to increase dimensionless fundamental frequencies of the segmented beams, $\widehat{\omega}$, above the one given in table 1 for the one-segmented half-span beams and corresponding two-segmented whole-span beams with $\mathrm{cc}$ boundary conditions and thus, the results for these cases are not tabulated in tables 4 and 5. A similar conclusion was made in the study dealing with the column buckling under cc boundary conditions in [22]. From the present study, it can be concluded that this is the case for the pp end conditions. In this case, the results showed that all segments of the beams have equal length and each segment has equal
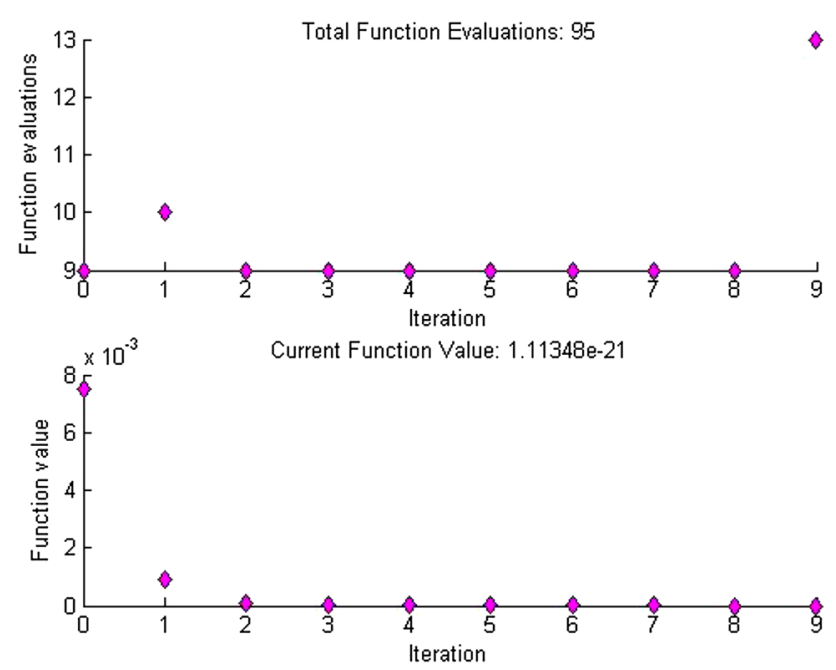

Figure 3. Total function evaluations and objective function value for the two-segmented beam with clamped-free end condition. volume fraction of the materials, that is $V_{A}=V_{B}=50 \%$. Moreover, it is observed from tables 4 and 5 that there is a small difference between design vectors for the half-span beams and corresponding whole-span ones. However, $\widehat{\omega}$ and corresponding gains for these cases are seen to be the same. This is not the case in reality if the more significant numbers are used. Nevertheless, it can be said that the differences between them can be regarded to be in the tolerable levels in view of production aspects.

On the other hand, in figures 3-5, the total function evaluations and the objective function versus iteration numbers are presented only for the cantilevered boundary condition. Other end conditions are not presented. Only their values are tabulated in tables 2, 3, 4 and 5. As expected, the number of total function evaluations and

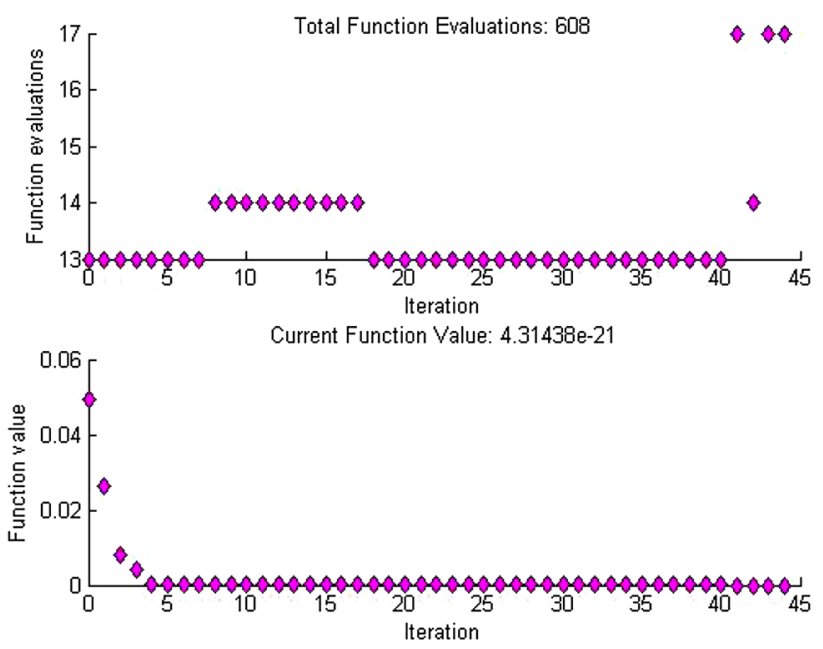

Figure 4. Total function evaluations and objective function value for the three-segmented beam with clamped-free end condition.
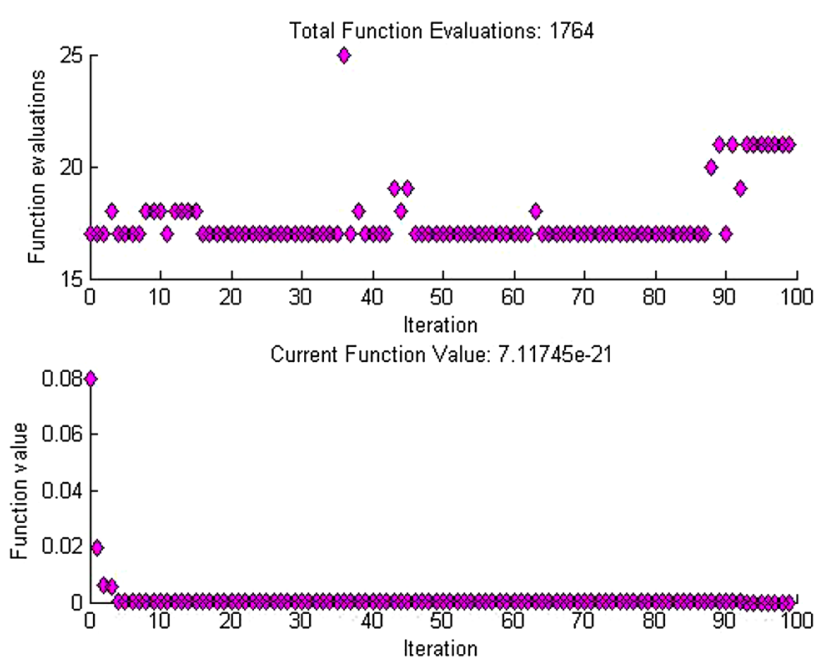

Figure 5. Total function evaluations and objective function value for the four-segmented beam with clamped-clamped end condition. 
iteration number increased for the cases with higher segment numbers due to increasing design space. This is the case for all other end conditions.

\section{Conclusion}

In this study, optimal design of the transversely vibrating Euler-Bernoulli beams segmented in the longitudinal direction under different end conditions is discussed and it is aimed to maximize the fundamental natural frequencies of the beams while keeping the total length and total mass of the optimized beams the same as those of the baseline design beam structures. The following results can be obtained:

- As mentioned in [14], selecting an objective function by taking the square of the characteristic equations ensures that the minimum would be around zero depending on the tolerance values. This is also the case for the present study. Hence, it is concluded that solving an eigenvalue problem, i.e., frequency equations, to obtain exact natural frequencies is identical to searching the design variables minimizing the objective functions and satisfying length and mass constraints.

- It is confirmed that for all boundary conditions, the fundamental natural frequency of the multi-segmented beams increases when compared with those of the onesegment uniform beams. An increase in the segment number results in an increase in the natural frequency values. At the same time, it should not be forgotten that the manufacturing cost will increase. Therefore, the designer or engineer should determine the beam configurations according to his/her initial design requirements.

- Among the end conditions, maximum increment in the fundamental natural frequency is more pronounced for the case of cc boundary condition. The fundamental natural frequency of the three-segment half-span and corresponding six-segment whole-span beams fixed at both ends is about 26.2500, which represents $17.3274 \%$ optimization gain. The structure with $\mathrm{cc}$ end conditions has higher stiffness, which in turn results in higher natural frequencies. Hence, the reason for observing the maximum increase in frequency of the beam with fixed-fixed boundary conditions can be attributed to this fact.

- It is also confirmed that there is no way to increase $\widehat{\omega}$ above the one given in table 1 for the one-segmented halfspan and corresponding two-segmented whole-span beam structures with cc boundary conditions. A similar conclusion is made for the column buckling problems considering cc end conditions in [22]. It is discerned from the present study that this is also the case for the simply supported end conditions.

On the other hand, the number of total function evaluations increased for the beam structures having higher segment numbers due to the increased design search spaces.
Finally, it can be said that this study is aimed to give some insight to the engineers/designers during their design stages.

\section{List of symbols}

$Y \quad$ deflection of the beam

$x \quad$ axial coordinate

$N_{s} \quad$ number of segments

$E_{k} \quad$ Young's modulus of the $k^{\text {th }}$ segment

$I_{k} \quad$ moment of inertia of the $k^{\text {th }}$ segment

$A_{k} \quad$ cross-section of the $k^{\text {th }}$ segment

$\rho_{k} \quad$ density of the $k^{\text {th }}$ segment

$L_{k} \quad$ length of the $k^{\text {th }}$ segment

$E \quad$ Young's modulus of the one-segmented beam

I moment of inertia of the one-segmented beam

$A$ cross-section of the one-segmented beam

$L \quad$ total length of the beam

$\rho$ density of the one-segmented beam

$\omega$ natural frequency of the beam

$\hat{\omega}$ dimensionless natural frequency of the beam

$\widehat{Y}$ dimensionless displacement

$\theta \quad$ slope

$\widehat{M}$ dimensionless bending moment

$\widehat{F} \quad$ dimensionless shear force

$M$ total mass of the baseline beam

$M_{s}$ total mass of the optimized segmented beam

$V \quad$ volume fraction of the materials

\section{References}

[1] Han S M, Benaroya H and Wei T 1999 Dynamics of transversely vibrating beams using four engineering theories. $J$. Sound Vib. 225: 935-988

[2] Maalawi K Y and El Chazly N M 2005 On the optimal design of beams in bending vibrations. J. Eng. Appl. Sci. 52: 889-903

[3] Barik M 2013 Higher modes natural frequencies of stepped beam using spectral finite elements. MS Thesis, National Institute of Technology, Rourkela, Odisha, India

[4] Lake S M and Mikulas M M 1991 Buckling and vibration analysis of a simply supported column with a piecewise constant cross section. NASA Technical Paper 3090, pp. $1-11$

[5] Kukla S and Rychlewska J 2013 Free vibration analysis of functionally graded beams. J. Appl. Math. Comput. Mech. 12: $39-44$

[6] Kukla S and Rychlewska J 2014 Free vibration of axially functionally graded Euler-Bernoulli beams. J. Appl. Math. Comput. Mech. 13: 39-44

[7] Li Q S, Fang J Q and Jeary A P 2000 Free vibration analysis of cantilevered tall structures under various axial loads. Eng. Struct. 22: 525-534 
[8] Goupee A J and Vel S S 2006 Optimization of natural frequencies of bidirectional functionally graded beams. Struct. Multidisc. Optim. 32: 473-484

[9] Kai-yuan Y, Xiao-hua T and Zhen-yi J 1992 General analytic solution of dynamic response of beams with nonhomogeneity and variable cross-section. Appl. Math. Mech. Engl. Ed. 13: 779-791

[10] Zhou D and Ji T 2006 Dynamic characteristics of a beam and distributed spring-mass system. Int. J. Solids Struct. 43: 5555-5569

[11] Maalawi K Y 2009 Optimization of elastic columns using axial grading concept. Eng. Struct. 31: 2922-2929

[12] Li Q S 2003 Effect of shear deformation on the critical buckling of multi-step bars. Struct. Eng. Mech. 15: 71-81

[13] Abbas L K and Rui X 2014 Free vibration characteristic of multilevel beam based on transfer matrix method of linear multibody systems. Adv. Mech. Eng. https://doi.org/10.1155/ 2014/792478, pp. $1-16$

[14] Alkan V 2015 Optimum buckling design of axially layered graded uniform columns. Mater. Test. 57: 474-480

[15] Alkan V 2015 Critical buckling load optimization of the axially graded layered uniform columns. Struct. Eng. Mech. 54: 725-740
[16] Topal U and Uzman U 2011 Frequency optimization of laminated skewed open cylindrical shells. Sci. Eng. Compos. Mater. 54: 139-144

[17] Topal U and Ozturk T H 2014 Buckling load optimization of laminated plates via artificial bee colony algorithm. Struct. Eng. Mech. 52: 755-765

[18] Topal U, Dede T and Ozturk T H 2017 Stacking sequence optimization for maximum fundamental frequency of simply supported antisymmetric laminated composite plates using teaching-learning-based optimization. KSCE J. Civ. Eng. 21: 2281-2288

[19] Singiresu S R 2013 Engineering optimization, theory and practice, 3rd enlarged ed. New Delhi: New Age International Publisher

[20] Venkataraman P 2002 Applied optimization with Matlab ${ }^{\circledR}$ programming. New York: John Wiley \& Sons, Inc.

[21] Matlab Help Documentation 2009 Matlab Optimization Toolbox. Massachusetts: Documentation Center, The MathWorks Inc.

[22] Maalawi K Y 2002 Buckling optimization of flexible columns. Int. J. Solids Struct. 39: 5865-5876 